\title{
El Foro Regional de la ASEAN : ¿un camino seguro hacia el multilateralismo para la seguridad en Asia Pacífico?
}

\section{$\mathrm{I}$} ntroducción

Los países de la región Asia Pacífico han experimentado, con escasas excepciones, un crecimiento sin precedentes durante las últimas décadas. Esto ha convertido a la zona en la más dinámica en términos de desempeño económico y, para muchos, en el centro de gravedad de la actividad económica mundial. Sin embargo, lo anterior también ha traído aparejados cambios en las relaciones de poder, que pueden desembocar en conflictos inesperados, lo cual es más probable dada la notable diversidad cultural, étnica, religiosa e histórica, sumada a la situación en la que conviven países muy grandes, muy pequeños y con diferentes grados de desarrollo.

Por otra parte, existe la percepción de que "Asia Pacífico ejemplifica exactamente la 'sociedad anárquica' de Hedley Bull, esto es, que los Estados compiten por poder e influencia en una arena internacional aún relativamente libre de coacción multilateral". ${ }^{1}$ Mientras tanto, otros ven que los mecanismos institucionales existentes en la región están en sus etapas primitivas, tales como el Foro Regional de la ASEAN $^{2}$ (ARF, por sus siglas en inglés) ${ }^{3}$ y el Consejo para la Cooperación en Seguridad de Asia Pacífico (CSCAP). ${ }^{4}$

En el presente trabajo se hará una evaluación, que no pretende ser exhaustiva, sobre las posibilidades de que el ARF sea el camino indicado para promover y fortalecer la seguridad y estabilidad regional en un marco multilateral. A tal efecto debemos tomar en cuenta que "el multilateralismo es una forma de cooperación internacional particularmente exigente. Éste requiere un fuerte sentido de

* Investigador del Departamento de Estudios del Pacífico de la Universidad de Guadalajara.

ORCID http://orcid.org/0000-0003-0344-6895 identidad colectiva además de intereses compartidos". 5

\section{Antecedentes}

En el caso de la región Asia Pacífico, el concepto del multilateralismo para contemplar cuestiones de seguridad, surgió sólo después del fin de la Guerra Fría. De este modo, la necesidad de un enfoque multilateral para el manejo de la seguridad en Asia Pacífico es vista más como el resultado de los cambios y alteraciones en la estructura de poder dentro de la región vis-àvis Japón y China, así como del derrumbe experimentado por el sistema imperante durante la Guerra fría. ${ }^{6}$ Sin embargo, otros factores a tomar en cuenta son que el dinamismo de la región y la intención de mantenerlo, hacían imperativo contar con una instancia que permitiera "construir confianza y patrones de cooperación, no sólo entre viejos amigos, sino [también] entre viejos adversarios.”7

Así, diversas propuestas surgieron para la creación de un mecanismo que permitiera mantener un orden más estable en la región Asia Pacífico. Entres éstas se encuentran las de Australia, Canadá y Japón, no sin ciertas reticencias por parte de algunos miembros de la ASEAN, que mantenían sus reservas acerca de la factibilidad y operatividad de una instancia regional que contemplara los asuntos sobre seguridad al estilo europeo. A pesar de esto, la ASEAN decidió sumarse, más que resistirse, a las preocupaciones mostradas con respecto al tema de la seguridad regional, no sin pronunciarse por la formación de un organismo independiente, sin la interferencia de los poderes externos a la región. Esto tendió a cambiar una vez que se llegó a la conclusión de que, para hacer viables los objetivos del ARF, se tenían que considerar dos partes: por un lado, los países del sudeste de Asia deberían promover 
sociedades estables y practicar la automoderación con sus vecinos, y, por otro, los grandes poderes externos (Estados Unidos, China, Japón, Rusia y la Unión Europea) deberían apoyar la estabilidad regional practicando la no interferencia. ${ }^{8}$

Ahora bien, aunque la creación del ARF respondió en gran parte a las propuestas externas a la ASEAN, el primero tendría que funcionar con base en las características distintivas de este último, en lo que los proponentes estuvieron de acuerdo. Lo anterior se reflejó en la adopción del Tratado de Amistad y Cooperación en el Sudeste de Asia, como el principal código de conducta para gobernar las relaciones entre los Estados y el único instrumento diplomático para forjar la confianza, la diplomacia preventiva y la cooperación política y de seguridad en Asia Pacífico. ${ }^{9}$ Así, el ARF se constituyó en julio de 1994 cuando, de acuerdo con la declaración de Singapur en 1992, tuvieron su primera reunión los ministros de relaciones exteriores de los entonces países miembros. ${ }^{10}$ Considerada en sí misma una reunión histórica, "significó la apertura de un nuevo capítulo de paz, estabilidad y cooperación para el sudeste de Asia." 11

\section{El ARF y la seguridad multilateral en Asia Pacífico}

Una vez que se creó el ARF, se cumplieron, cuando menos, dos objetivos muy claros: primero, involucró a China otorgándole un papel en el mantenimiento de la estructura de seguridad regional, y, segundo, integró a Estados Unidos en la nueva institución regional. Lo anterior y el diseño de la nueva estructura de seguridad dejaron en claro que los miembros de la ASEAN identificaron su propia seguridad con la participación de los poderes externos a ésta. ${ }^{12}$ Sin embargo, también mostró la disposición de Japón, Estados Unidos y China, principalmente, de apoyar un tipo de institución multilateral sobre aspectos de seguridad en Asia Pacífico.

La propuesta del gobierno japonés en julio de 1991, durante la Conferencia Post-ministerial de la ASEAN, de iniciar un diálogo sobre seguridad multilateral, representó un cambio radical de la política reactiva de Japón hacia la seguridad regional y fue la primera vez, desde el fin de la Segunda Guerra Mundial, que éste propusiera por sí mismo una iniciativa sobre seguridad regional, sin el apoyo de Estados Unidos. Dicha propuesta, además, contribuyó al cambio rápido de la actitud hostil por parte del gobierno de Estados Unidos hacia los arreglos sobre seguridad multilateral en Asia Pacífico. ${ }^{13} \mathrm{La}$ constante en esta región había sido la preferencia de Estados Unidos por los acuerdos bilaterales, puesto que no creían que los Estados del sudeste de Asia pudieran hacerse cargo del aumento de la influencia que una institución multilateral ofrecería, ni había la sensación de que dichos Estados merecieran tal estructura multilateral. ${ }^{14}$ Pero esto empezó a cambiar hacia finales de los años ochenta con el fin de la Guerra Fría, además de que la creciente tolerancia e interés de Estados Unidos hacia el ARF refleja este cambio de política. Cabe aclarar, sin embargo, que dicho cambio no implica la creencia en que las alianzas bilaterales tengan que suprimirse sino que, por el contrario, éstas tienen mucho que aportar a la puesta en práctica de una estructura multilateral viable..$^{15}$

El enfoque de China hacia los arreglos multilaterales, por otra parte, mantiene una marcada preferencia por el comportamiento unilateral. No obstante, su decisión de formar parte del ARF, además de que significó un fuerte 
impulso a éste, "es indicio de un ligero cambio en la política de seguridad de China, que refleja su sensibilidad a por lo menos algunas sugerencias internacionales, lo que bien puede augurar la voluntad de China para considerar dar un mayor apoyo al ARF o a otros arreglos multilaterales en el futuro". ${ }^{16}$ Esto es así quizá debido a que, aparentemente, el liderazgo chino está contento con la estrategia del equilibrio de poder seguida por parte de la ASEAN, la cual ayuda a prevenir que cualquier poder más grande, incluyendo a Estados Unidos, llegue a dominar la región Asia Pacífico, lo que sin duda va de acuerdo con los intereses chinos, puesto que China no tiene la capacidad y, por lo tanto, la intención de llegar a ser el poder predominante en los asuntos regionales. ${ }^{17}$

Ahora bien, la formación del ARF recuerda el enfoque cauteloso del establecimiento de consensos y los acuerdos bilaterales, con el fin de preparar el terreno para acceder a una conducta multilateral. Esto refleja en gran parte la preferencia asiática, a diferencia de la occidental, de no forzar un enfoque legal sobre los acuerdos, sino proceder paso a paso y permitir que dichos acuerdos evolucionen por sí mismos antes de su institucionalización, si ésta fuera necesaria. Así, el uso del formato más simple, un foro, y el enfoque evolutivo gradual con el que se previó la creación del ARF, representaron un acoplamiento de las preocupaciones acerca de la institucionalización de éste y del papel de la ASEAN en él. El mencionado enfoque evolutivo se puede apreciar en la concepción de las tres etapas por las cuales debe transitar dicho foro. La primera tiene que ver con la promoción de medidas para fomentar la confianza entre las partes; la segunda se refiere al desarrollo de mecanismos para llevar a cabo la diplomacia preventiva, y la tercera permitiría el desarrollo de mecanismos de resolución de conflictos. ${ }^{18}$

Medidas para fomentar la confianza

En razón de lo apremiante de algunas situaciones políticas y de seguridad en la región, había la necesidad de establecer un programa que ayudara a mejorar la confianza entre los Estados de la región Asia Pacífico. Este objetivo es uno de los más importantes del $\mathrm{ARF}$, puesto que se reconoce, de entrada, que, en comparación con las estructuras de cooperación en aspectos de seguridad prevalecientes durante la Guerra Fría, este foro opera sobre bases diferentes. Así, no se sustenta en la amenaza o el uso de la fuerza ni tiene una función de contención o de disuasión, cuya característica es la opacidad, sino en la confianza mutua y la transparencia y enfatiza los procesos más que las estructuras organizativas y de comandos. De este modo, las medidas para fomentar la confianza fueron identificadas como un aspecto fundamental para este nuevo enfoque de cooperación multilateral en aspectos de seguridad. Esto está apoyado en la premisa de que entre los miembros del foro no hay enemigos específicos. Lo anterior no significa que se eliminen los problemas de manera automática, puesto que aún con la operación apropiada del foro puede haber desacuerdos y tensiones, sino que estos pueden ser abordados en un clima de transparencia y confianza entre las partes involucradas, con el cobijo del ARF.

La incorporación de Corea del Norte, en julio de 2000, a la membresía del ARF, hizo frente a uno de los principales factores potenciales de desestabilización de la región Asia Pacífico. Esto trajo al seno del foro un problema difícil de resolver, pero no podía seguir dejándose de lado, pues ignorarlo era como esconder la cabeza en la tierra a pesar de las llamadas de atención sobre el tema. ${ }^{19}$ Aunque la sola admisión no resuelve el problema, como se ha dejado ver en las fechas posteriores, por lo menos abre la posibilidad de que "se promueva la amistad y la armonía y cooperar para la paz y la seguridad en la región Asia Pacífico", ${ }^{0}$ como lo expresó el propio ministro de relaciones exteriores de Corea del Norte, Paek Nam Sun, en su discurso preparado para la ceremonia de admisión. Sin embargo, el asunto se ve cada vez más complicado debido a la inclusión de Corea del Norte en la lista de países que componen "el eje del mal”, denominado así por parte de Estados Unidos en su lucha contra el terrorismo, y a la capacidad y disposición de la primera de 
producir armas nucleares, aun en contra de los acuerdos multilaterales alcanzados sobre el tema.

Otro avance, aunque relativo, es la publicación del Panorama Anual de Seguridad del ARF, del cual se han hecho tres documentos (2000-2002) en el que se asientan las principales preocupaciones de cada uno de los participantes. Cabe mencionar que estos documentos se integran con las aportaciones de cada uno de los países miembros de manera voluntaria y se publican sin mediar ningún trabajo de edición. La intención es compartir visiones sobre el ambiente estratégico inmediato de la región, así como contribuir a la promoción de la confianza y el entendimiento entre los participantes, lo que, a su vez, fomente la paz, seguridad y prosperidad en la región Asia Pacífico. ${ }^{21}$

\section{La diplomacia preventiva}

En el contexto del ARF la diplomacia preventiva significa el impulso de acciones para prevenir las disputas que puedan surgir entre las partes; evitar que las existentes se conviertan en conflictos, y limitar la ampliación de éstos cuando, a pesar de todo, se presenten. Sin embargo, esta fase no se puede llevar a cabo sin un avance sustantivo en los objetivos de la primera etapa, puesto que las negociaciones deben respaldarse en la existencia de un alto grado de confianza, de lo contrario es casi imposible que se llegue a acuerdos para el establecimiento de mecanismos que puedan ser percibidos de manera desfavorable por alguna de las partes.

De esta manera, la ventaja de la diplomacia preventiva, dentro de un grupo que busca la cooperación multilateral para la seguridad, es que el foro entero permite contar con un efecto amortiguador para las tensiones y posibles conflictos regionales. Además, tal diplomacia propicia también un fortalecimiento de las relaciones bilaterales en tanto que se estipulan los medios adecuados para tratar los asuntos a través de métodos pacíficos no militares. Así, uno de los métodos usados para lograr avances en la diplomacia preventiva ha sido el Tratado de Amistad y Cooperación, que sirve como un código de conducta entre los Estados miembros y funciona también como un pacto de no agresión, estimulando la resolución pacífica de los conflictos. Finalmente, el logro de esta meta podría ser una buena señal de que el ARF está listo para afrontar exitosamente cualquier desafío a la paz y la seguridad de la región Asia Pacífico. ${ }^{22}$

\section{Resolución de conflictos}

Dado que el establecimiento de mecanismos de resolución de conflictos no está contemplado por el ARF a corto plazo, la tarea de determinarlos es una meta final que todos los participantes deberían tener en cuenta, en tanto que dichos mecanismos marcarán la pauta de desarrollo del foro que lo consolide como medio para el mantenimiento de la paz y estabilidad regional.

De lo que pase en este aspecto depende en gran parte lo que resulte al final de cuentas el $\mathrm{ARF}$, pues esta delicada etapa indicará hacia dónde se dirige. La prueba más difícil será la evolución hacia la recomendación de medidas que impliquen decisiones políticas delicadas. Incluso se podría pensar en que estos desafíos obliguen a una reconsideración de la estructura inicial con la que fue concebido el foro, lo cual ha sido ya sugerido y va en el sentido de que se incorpore a los jefes de Estado de cada uno de los países participantes, de modo similar a como se suscitó en el foro de Cooperación Económica Asia Pacífico (APEC), con el fin de darle un gran impulso a su estatura y autoridad. Además, al igual que en el foro de APEC, se argumenta la necesidad de abrir la conducción del ARF para que los demás países participantes, no miembros de la ASEAN, se puedan involucrar y comprometer más en la consolidación del foro. ${ }^{23}$

\section{Conclusiones}

Después de poco más de ocho años de vida, el ARF ha navegado por aguas tranquilas, en comparación con lo que puede requerir su fortalecimiento a fin de cumplir con los objetivos para los que fue concebido: preservar y ampliar 
la paz, estabilidad y prosperidad de la región Asia Pacífico. Esto no quiere decir que no haya cumplido con los objetivos durante el trayecto que su propia concepción evolutiva le ha impuesto, sino que la etapa definitiva puede significar su derrumbe, a pesar del camino recorrido hasta hoy.

El momento de su creación significó, por sí mismo, un éxito al sentar alrededor de la misma mesa a los principales actores regionales para tratar aspectos sobre seguridad en su ámbito. Esto no es un logro despreciable si se toman en cuenta las disparidades de todo tipo existentes entre los involucrados y la escasa o nula experiencia de comportamiento multilateral en cuestiones de seguridad en la zona como un todo.

Las dos etapas por las que ha transitado, o en las que se encuentra si se toma en cuenta que no son etapas que terminen de una manera definitiva, han permitido al ARF subsanar, en cierta medida, esa escasa experiencia y establecer las bases sobre las cuales construir, en cimientos más firmes, la estructura definitiva que habrá de regir las relaciones entre los Estados, con el fin de disminuir el grado de anarquía imperante en la zona, a lo que contribuye también, en cierta medida, cada una de las etapas concebidas para la evolución del $\mathrm{ARF}$.

Hasta ahora, pues, la función que ha desempeñado el ARF ha sido contribuir a la construcción paulatina de un sentido de responsabilidad compartida entre los Estados para el mantenimiento de la paz y la estabilidad regional. De esta manera, se ha avanzado en el reconocimiento de una estrecha interdependencia, lo que ha permitido, a su vez, la identificación de intereses comunes $o$, al menos, complementarios, y podría, en última instancia, conducir al surgimiento de un sentimiento de identidad regional.

Sin embargo, se debe reconocer que la parte fuerte del proceso está por venir y que su conducción requiere actuar con mucha imaginación y no creer que el éxito esté garantizado, por lo que de ninguna manera se puede dar por hecho que la consolidación del ARF sea algo ya consumado. Así, falta todavía navegar aguas turbulentas en el camino a su cimentación como un foro que realmente conduzca a la cooperación multilateral efectiva en materia de seguridad en la región AsiaPacífico.

\section{Notas}

1 Ron Huisken, "Civilizing the Anarchical Society: Multilateral Security Process in the Asia Pacific", Contemporary Southeast Asia, vol. 24, núm., 2, agosto de 2002 , p. 188.

2 Asociación de Naciones del Sudeste de Asia (ASEAN, por sus siglas en inglés).

3 En adelante las siglas que se utilicen corresponderán al nombre en inglés, salvo que se haga aclaración en otro sentido.

4 Ki-Jung Kim y Yongho Kim, "Alliance Versus Governance: Theoretical Debates Regarding Security of Northeast Asia”, Pacific Focus, vol. XVII, núm. 1, primavera de 2002, p. 11.

5 Christopher Hemmer y Peter J. Katzenstein, "Why is There No Nato in Asia? Collective Identity, Regionalism, and the Origins of Multilateralism", International Organization, vol. 56, núm. 3, verano de 2002, pp. 575-576.

6 Shankari Sandararaman, "The ASEAN Regional Forum: Reassessing Multilateral Security in the Asia Pacific", Strategic Analysis, 22, núm. 4 (julio de 1998), pp. 655-665. Disponible en versión electrónica en http:/ /www.idsa-india.org/an-jul-11html.

7 Michael Vatikiotis apud Michael Antolik, "The ASEAN Regional Forum: The Spirit of Constructive Engagement", Contemporary Southeast Asia, vol. 16, núm. 2, septiembre de 1994, p. 118.

8 Dagoberto Amparo, "El Foro Regional de ASEAN", México y la Cuenca del Pacífico, vol. 2, núm 8, octubrediciembre de 1999, pp. 55-59.

9 Secretariado de ASEAN, The ASEAN Regional Forum: A Concept Paper, en http://www.aseansec.org/3693.htm.

10 Estos eran: Australia, Brunei, Canadá, China, Corea del Sur, Estados Unidos, Filipinas, Indonesia, Japón, Laos, Malasia, Mongolia, Nueva Zelanda, Papua Nueva Guinea, Rusia, Singapur, Tailandia y Vietnam. Camboya se incorporó en 1995, durante la segunda reunión del ARF; India y Myanmar lo hicieron en 1996; Mongolia en 1998, y Corea del Norte se sumó en el año 2000.

11 Secretariado de ASEAN, Chairman's Statement The First ASEAN Regional Forum, en http://aseansec.org/ 2879.htm.

12 Tobias Nischalke, "Does ASEAN Measure Up? PostCold War Diplomacy and the Idea of Regional Community", The Pacific Review, vol. 15, num. 1, 2002, p. 100. Véase también Michael Antolik, op. cit., pp. 117136, y Joseph Y. S. Cheng, "China's ASEAN Policy in 
the 1990's: Pushing for Regional Multipolarity", Contemporary Southeast Asia, vol. 21, núm. 2, agosto de 1999, pp. 176-204.

13 Nobuo Okawara y Peter J. Katzenstein, "Japan and Asian-Pacific Security: Regionalization, Entrenched Bilateralism and Incipient Multilateralism", The Pacific Review, vol. 14, núm., 2, 2001, p. 177.

14 Christopher Hemmer y Peter J. Katzenstein, op. cit., p. 588.

15 Chin Kin Wah y Pang Eng Fong, Relating the U. S.Korea and U. S.-Japan Alliances to Emerging Asia Pacific Multilateral Processes: An ASEAN Perspective, Project Discussion Papers del Asia/Pacific Research Center, Institute for International Studies, Stanford University, marzo de 2000, p. 13. Los documentos de esta serie están disponibles en versión electrónica en http://www.stanford.edu/group/APARC. Véase también Douglas Paal, Nesting the Alliances in the Emerging Context of Asia-Pacific Multilateral Processes: A U. S. Perspective, Project Discussion Papers del Asia/Pacific Research Center, Institute for International Studies, Stanford University, julio de 1999.

16 Nobuo Okawara y Peter J. Katzenstein, op. cit., p. 179.

17 Joseph Y. S. Cheng, op. cit., p. 185.

18 Dagoberto Amparo, op. cit., p. 57.

19 John Garofono, "Flexibility or Irrelevance: Ways Forward for the ARF", Contemporary Southeast Asia, vol. 21, num. 1, abril de 1999, pp. 74-94. Véase también Chin Kin Wah y Pang Eng Fong, op. cit., p. 13.

20 DPRK Joins ARF, Pledges to Promote Regional Security, Cooperative Relations, en http://www.korea-np.co.jp/pk.

21 ASEAN Regional Forum, "Foreword", Annual Security Outlook 2002, p. 2. Los tres documentos anuales que hasta la fecha se han publicado están disponibles en versión electrónica en: http://www.aseansec.org./amm/ aso/.

22 Shankari Sandararaman, op. cit., pp. 655-665.

23 Ron Huisken, op. cit., pp. 199-200. 